Canad. J. Math. Vol. 57 (2), 2005 pp. 328-337

\title{
On a Conjecture of Birch and Swinnerton-Dyer
}

\author{
Wentang Kuo and M. Ram Murty
}

Abstract. Let $E / \mathbb{O}$ ) be an elliptic curve defined by the equation $y^{2}=x^{3}+a x+b$. For a prime $p$, $p \nmid \Delta=-16\left(4 a^{3}+27 b^{2}\right) \neq 0$, define

$$
N_{p}=p+1-a_{p}=\left|E\left(\mathbb{F}_{p}\right)\right| .
$$

As a precursor to their celebrated conjecture, Birch and Swinnerton-Dyer originally conjectured that for some constant $c$,

$$
\prod_{p \leq x, p \nmid \Delta} \frac{N_{p}}{p} \sim c(\log x)^{r}, \quad x \rightarrow \infty .
$$

Let $\alpha_{p}$ and $\beta_{p}$ be the eigenvalues of the Frobenius at $p$. Define

$$
\tilde{c}_{n}= \begin{cases}\frac{\alpha_{p}^{k}+\beta_{p}^{k}}{k} & n=p^{k}, p \text { is a prime, } k \text { is a natural number, } p \nmid \Delta . \\ 0 & \text { otherwise. }\end{cases}
$$

and $\tilde{C}(x)=\sum_{n \leq x} \tilde{c}_{n}$. In this paper, we establish the equivalence between the conjecture and the condition $\tilde{C}(x)=\mathbf{o}(x)$. The asymptotic condition is indeed much deeper than what we know so far or what we can know under the analogue of the Riemann hypothesis. In addition, we provide an oscillation theorem and an $\Omega$ theorem which relate to the constant $c$ in the conjecture.

\section{Introduction}

Let $E /(\mathbb{O})$ be an elliptic curve, defined as

$$
y^{2}=x^{3}+a x+b
$$

For a prime $p, p \nmid \Delta=-16\left(4 a^{3}+27 b^{2}\right) \neq 0$, define

$$
N_{p}=p+1-a_{p}=E\left(\mathbb{F}_{p}\right)
$$

As a precursor to their celebrated conjecture, Birch and Swinnerton-Dyer formulated the following:

Conjecture ([2] Birch and Swinnerton-Dyer) For some constant c,

$$
\prod_{p \leq x, p \nmid \Delta} \frac{N_{p}}{p} \sim c(\log x)^{r}, \quad x \rightarrow \infty .
$$

Received by the editors March10, 2003; revised February 3, 2004.

Research of the second author was partially supported by an NSERC grant.

AMS subject classification: Primary: 11M41; secondary: 11M06.

(C)Canadian Mathematical Society 2005. 
We use B-SD as the abbreviation of this Birch and Swinnerton-Dyer conjecture. Now consider the $L$-function $L_{E}(s)$ attached to $E$ defined as follows:

$$
\begin{aligned}
L_{E}(s): & =\prod_{p \nmid \Delta}\left(1-a_{p} p^{-s}+p^{-2 s}\right)^{-1} \times \prod_{p \mid \Delta} l_{p}(E, s)^{-1} \\
& =\prod_{p \nmid \Delta}\left(1-\frac{\alpha_{p}}{p^{s}}\right)^{-1}\left(1-\frac{\beta_{p}}{p^{s}}\right)^{-1} \times \prod_{p \mid \Delta} l_{p}(E, s)^{-1},
\end{aligned}
$$

where $l_{p}(E, s)$ is a certain polynomial in $p^{-s}$ with the property that $l_{p}(E, 1) \neq 0$. By the work of Wiles [10], and Breuil, Conrad, Diamond and Taylor [3], $L_{E}(s)$ extends to an entire function and satisfies a functional equation relating $L_{E}(s)$ to $L_{E}(2-s)$.

Let

$$
\tilde{L}_{E}(s):=\prod_{p \nmid \Delta}\left(1-a_{p} p^{-s}+p^{-2 s}\right)^{-1} .
$$

In [5], Goldfeld examined the consequences of the B-SD conjecture. He proved that the constant $c$ is the asymptotic formula is $\sqrt{2} / \tilde{L}_{E}(1)$. He also he proved that it implies the Riemann hypothesis for $L_{E}(s)$, namely that all the non-trivial zeros of $L_{E}(s)$ lie on $\Re(s)=1$.

We prove the following results below. Define the sequences $c_{n}, \tilde{c}_{n}$ and their summatory functions as follows:

$-\log \tilde{L}_{E}(s+1)=\sum_{n \in \mathbb{N}} \frac{c_{n}}{n^{s}}, \quad-\log \tilde{L}_{E}(s)=\sum_{n \in \mathbb{N}} \frac{\tilde{c}_{n}}{n^{s}}, \quad C(x)=\sum_{n \leq x} c_{n}, \quad \tilde{C}(x)=\sum_{n \leq x} \tilde{c}_{n}$.

Theorem (Theorems 2 and 3) The B-SD conjecture is true if and only if $\tilde{C}(x)=\mathbf{o}(x)$.

Theorems 2 and 3 establish the equivalence of the B-SD conjecture with the behavior of a familiar sum appearing in analytic number theory. The latter sum has been analyzed in the classical context of the Riemann zeta function by Montgomery [8] and one would expect similar behavior for $L_{E}(s)$. Assuming this, it becomes transparent that the B-SD conjecture is very likely to be true and very unlikely that it will ever be proved in the near future.

In addition to this, we apply an oscillation theorem of Landau to "explain" some observed oscillatory behavior of the products occurring in the B-SD conjecture. More precisely, assuming the B-SD conjecture, in Lemma 1, we show that

$$
C(x)=-r \log \log x+A+R(x),
$$

where $A$ is a constant and $R(x)=\mathbf{o}(1)$. Then

Theorem (Theorem 4 and Proposition 5) Assume the B-SD conjecture. Then $R(x)$ oscillates. Furthermore, for any $\epsilon>0, R(x)$ is $\Omega\left(1 /(\log x)^{1+\epsilon}\right)$.

After this work was done, K. Murty informed us that Keith Conrad [4] has also proved Theorem 3 in the more general context of $L$-functions with Euler products of $G L(n)$-type. His proof uses the explicit formula method. Our proof invokes a beautiful, little known theorem of Marcel Riesz [9] and is shorter than the one in [4]. Our approach can also be used to treat the general context. 


\section{Notations and Preparation}

Let $E /(\mathbb{O})$ be an elliptic curve, defined as

$$
y^{2}=x^{3}+a x+b
$$

For a prime $p, p \nmid \Delta=-16\left(4 a^{3}+27 b^{2}\right) \neq 0$, define

$$
N_{p}=p+1-a_{p}=\left|E\left(\mathbb{F}_{p}\right)\right|
$$

where $E\left(\mathbb{F}_{p}\right)$ is the set of solutions of the equation $y^{2}=x^{3}+a x+b$ in the finite field $\mathbb{F}_{p}$ together with the point at infinity.

To ease the notation, we use

$$
\prod^{\prime}=\prod_{p \nmid \Delta} \text { and } \sum^{\prime}=\sum_{p \nmid \Delta}
$$

Let $\alpha_{p}$ and $\beta_{p}$ be the eigenvalues of the Frobenius at $p$, and we have

$$
a_{p}=\alpha_{p}+\beta_{p}, \quad \alpha_{p} \cdot \beta_{p}=p .
$$

Therefore

$$
\begin{aligned}
N_{p} & =p+1-a_{p}=\frac{1}{p}\left(p^{2}+p-p \cdot a_{p}\right) \\
& =\frac{1}{p}\left(p^{2}-p\left(\alpha_{p}+\beta_{p}\right)+\alpha_{p} \cdot \beta_{p}\right)=\frac{1}{p}\left(p-\alpha_{p}\right)\left(p-\beta_{p}\right) .
\end{aligned}
$$

Thus, the product in the conjecture becomes

$$
\prod_{p \leq x}^{\prime} \frac{N_{p}}{p}=\prod_{p \leq x}^{\prime}\left(1-\frac{\alpha_{p}}{p}\right)\left(1-\frac{\beta_{p}}{p}\right) .
$$

Take $-\log$ on both sides:

$$
-\sum_{p \leq x}^{\prime} \log \left(1-\frac{\alpha_{p}}{p}\right)\left(1-\frac{\beta_{p}}{p}\right)=\sum_{p \leq x}^{\prime} \sum_{k=1}^{\infty} \frac{\alpha_{p}^{k}+\beta_{p}^{k}}{k p^{k}}
$$

Define

$$
C(x):=\sum_{p^{k} \leq x}^{\prime} \frac{\alpha_{p}^{k}+\beta_{p}^{k}}{k p^{k}}=\sum_{n \leq x} c_{n},
$$

where

$$
c_{n}= \begin{cases}\frac{\alpha_{p}^{k}+\beta_{p}^{k}}{k p^{k}} & n=p^{k}, p \text { is a prime, } k \text { is a natural number, }(p, \Delta)=1 \\ 0 & \text { otherwise. }\end{cases}
$$


Similarly, we define

$$
\tilde{C}(x):=\sum_{p^{k} \leq x}^{\prime} \frac{\alpha_{p}^{k}+\beta_{p}^{k}}{k}=\sum_{n \leq x} \tilde{c}_{n}
$$

where

$$
\tilde{c}_{n}=c_{n} \cdot n= \begin{cases}\frac{\alpha_{p}^{k}+\beta_{p}^{k}}{k} & n=p^{k}, p \text { is a prime, } k \text { is a natural number, } p \nmid \Delta, \\ 0 & \text { otherwise. }\end{cases}
$$

For instance, $\tilde{c}_{p}=a_{p}, \tilde{c}_{p^{2}}=\left(a_{p}^{2}-2 p\right) / 2, \ldots$, etc.

Now we consider the partial $L$-function $\tilde{L}_{E}(s)$ attached to $E$ defined as follows:

$$
\tilde{L}_{E}(s):=\prod^{\prime}\left(1-a_{p} p^{-s}+p \cdot p^{-2 s}\right)^{-1}=\prod^{\prime}\left(1-\frac{\alpha_{p}}{p^{s}}\right)^{-1}\left(1-\frac{\beta_{p}}{p^{s}}\right)^{-1} .
$$

We take the log of the $L$-functions:

$$
-\log \tilde{L}_{E}(s+1)=\sum_{p}^{\prime} \sum_{k \geq 1}^{\infty} \frac{\alpha_{p}^{k}+\beta_{p}^{k}}{k p^{(s+1) k}}=\sum_{n \in \mathbb{N}} \frac{c_{n}}{n^{s}}=\int_{1}^{\infty} \frac{1}{x^{s}} d C(x)=s \int_{1}^{\infty} \frac{C(x)}{x^{s+1}} d x
$$

and

$$
-\log \tilde{L}_{E}(s)=\sum_{p}^{\prime} \sum_{k \geq 1}^{\infty} \frac{\alpha_{p}^{k}+\beta_{p}^{k}}{k p^{s k}} .=\sum_{n \in \mathbb{N}} \frac{\tilde{c}_{n}}{n^{s}}=\int_{1}^{\infty} \frac{1}{x^{s}} d \tilde{C}(x)=s \int_{1}^{\infty} \frac{\tilde{C}(x)}{x^{s+1}} d x .
$$

Lemma 1 If we assume the B-SD conjecture, then

$$
C(x)=-r \log \log x+A+\mathbf{o}(1)
$$

where $A$ is a constant.

Proof Assuming the B-SD conjecture, we have

$$
\begin{aligned}
-r \log \log x-\log c+\log (1+\mathbf{o}(1)) & =\sum_{p \leq x}^{\prime} \sum_{k=1}^{\infty} \frac{\alpha_{p}^{k}+\beta_{p}^{k}}{k p^{k}}=\sum_{p^{k} \leq x} c_{p^{k}}+\sum_{p \leq x, x<p^{k}} c_{p^{k}} \\
& =C(x)+e(x),
\end{aligned}
$$

where $e(x)=\sum_{p \leq x, x<p^{k}} c_{p^{k}}$. All we need to do is to prove $e(x)=a+\mathbf{o}(1)$. We know

$$
\left|\alpha_{p}^{k}\right| \leq p^{\frac{k}{2}}, \quad\left|\beta_{p}^{k}\right| \leq p^{\frac{k}{2}} \Longrightarrow\left|\alpha_{p}^{k}+\beta_{p}^{k}\right| \leq 2 p^{\frac{k}{2}}
$$

Therefore, the sum $\sum_{p \leq x, x<p^{k}, 3 \leq k} c_{p^{k}}$ is absolutely convergent as $x$ goes to infinity. Hence, we can ignore this part. Define

$$
e_{2}(x):=\sum_{p \leq x} c_{p^{2}} .
$$


Now we concentrate on

$$
\tilde{e}_{2}(x):=e(x)-\sum_{\substack{p \leq x \\ x<p^{k} \\ 3 \leq k}} c_{p^{k}}=\sum_{\sqrt{x} \leq p \leq x} c_{p^{2}}=e_{2}(x)-e_{2}(\sqrt{x}) .
$$

By definition

$$
c_{p^{2}}=\frac{1}{2} \cdot \frac{\alpha_{p}^{2}+\beta_{p}^{2}}{p^{2}}=\frac{1}{2} \cdot \frac{a_{p}^{2}-2 p}{p^{2}}
$$

since $a_{p}^{2}-2 p=\alpha_{p}^{2}+\beta_{p}^{2}$. By the theory of the Rankin-Selberg convolution (see [7]), we have

$$
\sum \frac{a_{p}^{2}}{p^{s}} \sim \log \frac{1}{s-2} \Longrightarrow \frac{1}{2} \sum \frac{a_{p}^{2}}{p^{2 s}} \sim \frac{1}{2} \log \frac{1}{2(s-1)} \sim \frac{1}{2} \log \frac{1}{s-1} .
$$

This implies

$$
\frac{1}{2} \sum_{p^{2} \leq x} \frac{a_{p}^{2}}{p^{2}}=\frac{1}{2} \log \log x+B_{1}+\mathbf{o}(1)
$$

for some constant $B_{1}$. Therefore,

$e_{2}(x)=\frac{1}{2} \sum_{p \leq x} \frac{a_{p}^{2}-2 p}{p^{2}}=\frac{1}{2} \log \log x-\log \log x+c_{2}+\mathbf{o}(1)=-\frac{1}{2} \log \log x+B_{2}+\mathbf{o}(1)$,

where $B_{2}$ is a constant. Hence,

$$
\tilde{e}_{2}(x)=e_{2}(x)-e_{2}(\sqrt{x})=\frac{1}{2} \log \frac{1}{2}+\mathbf{o}(1) .
$$

This completes the proof.

Remark We have two remarks on this lemma.

1. In Goldfeld's paper [5], there is an extra $\sqrt{2}$ factor in his residue formula. In [4], K. Conrad demystifies this extra factor in the much more general context alluded to earlier. In our case, though we do not discuss it in our paper, it is clear that this extra factor comes from the constant $1 / 2 \cdot \log 1 / 2$ in the last equation of the proof of the previous lemma.

2. By the lemma above, we can get the equivalence between the B-SD conjecture

$$
\prod_{p \leq x}^{\prime} \frac{N_{p}}{p}=c(x)(\log x)^{r}, \quad c(x)=c+r(x), r(x)=\mathbf{o}(1),
$$

and the asymptotic formula

$$
C(x)=-r \log \log x+A+R(x), R(x)=\mathbf{o}(1) .
$$

The lemma gives us the direction from the conjecture to the asymptotic formula. The other direction is also true since by the lemma, the difference between $C(x)$ and $-\log \left(\prod_{p \leq x}^{\prime} N_{p} / p\right)$ is convergent as $x$ goes to infinity. 


\section{An o-theorem}

The main theorem of this paper is to establish the equivalence between the B-SD conjecture and a little o-condition. Let us start in one direction.

Theorem 2 Suppose that the B-SD conjecture is true, then

$$
\sum_{n \leq x} \tilde{c}_{n}=\mathbf{o}(x)
$$

Proof By partial summation,

$$
\sum_{n \leq x} \tilde{c}_{n}=\sum_{n \leq x} c_{n} \cdot n=x \cdot C(x)-\int_{3}^{x} C(t) d t+\mathbf{o}(1),
$$

where as usual $C(x)=\sum_{n \leq x} c_{n}$. By Lemma 1 we have

$$
C(x)=-r \log \log x+A+\mathbf{o}(1) .
$$

Therefore,

$$
\begin{aligned}
\sum_{n \leq x} \tilde{c}_{n} & =x \cdot C(x)-\int_{3}^{x} C(t) d t+\mathbf{o}(1) \\
& =x\{-r \log \log x+A+\mathbf{o}(1)\}-\int_{3}^{x}-r \log \log t+A+\mathbf{o}(1) d t+\mathbf{o}(1) \\
& =-r x \log \log x+c x-\left(\int_{3}^{x}-r \log \log t d t+c x+\mathbf{o}(x)\right) \\
& =-r x \log \log x+\int_{3}^{x} r \log \log t d t+\mathbf{o}(x)
\end{aligned}
$$

Hence, after an easy integration,

$$
\sum_{n \leq x} \tilde{c}_{n}=-r x \log \log x+r\left(x \log \log x+\mathbf{o}(x)+\mathbf{O}\left(\frac{x}{\log x}\right)\right)=\mathbf{o}(x) .
$$

Amazingly, the converse of the previous theorem is true. We need the following theorem of Riesz.

Theorem ([9, Satz I]; [6, Part 2 of the Theorem $]$ ) Assume that

$$
\lambda_{1}<\lambda_{2}<\cdots, \quad \lambda_{n} \rightarrow \infty, \quad B(y)=\sum_{\lambda_{n} \leq y} b_{n}=\mathbf{O}\left(e^{y}\right), \quad g(s)=\sum_{n \geq 1} b_{n} e^{-\lambda_{n} s} .
$$

Hence $g(s)$ is convergent for $\Re(s)>1$. Suppose that $g(s)$ is regular at $s=1$. If one assumes that

$$
B(y)=\mathbf{o}\left(e^{y}\right),
$$

then

converges.

$$
\sum_{n \geq 1} b_{n} e^{-\lambda_{n}}
$$


In addition, we require a little lemma.

Lemma If $\sum_{n \leq x} \tilde{c}_{n}=\mathbf{O}(x)$, then $L_{E}(s)$ has no zero $\Re(s)>1$.

Proof We need only consider $\tilde{L}_{E}(s)$. We know

$$
-\log \tilde{L}_{E}(s)=\sum \frac{\tilde{c}_{n}}{n^{s}}
$$

For $\Re(s)=\sigma>1$,

$$
\begin{aligned}
-\log \tilde{L}_{E}(s) & =\sum \frac{\tilde{c}_{n}}{n^{s}}=\int_{1}^{\infty} \frac{1}{x^{s}} d \tilde{C}(x) \\
& =s \int_{1}^{\infty} \frac{\tilde{C}(x)}{x^{s+1}} d x=\mathbf{O}\left(\int_{1}^{\infty} \frac{x}{x^{\sigma+1}} d x\right)=\mathbf{O}\left(\int_{1}^{\infty} \frac{1}{x^{\sigma}} d x\right) .
\end{aligned}
$$

$\log \tilde{L}_{E}(s)$ is regular for $\Re(s)>1$. Therefore, $\tilde{L}_{E}(s)$ has no zero for $\Re(s)>1$.

Now we are ready for our theorem.

Theorem 3 If $\sum_{n \leq x} \tilde{c}_{n}=\mathbf{o}(x)$, then the B-SD conjecture is true.

Proof By the lemma above, $\log \tilde{L}_{E}(s)$ is regular for $\Re(s)>1$. Let $r$ be such that

$$
-\log \tilde{L}_{E}(s)+r \log (s-1)=\sum \tilde{c}_{n} \cdot n^{-s}+r \log (s-1)
$$

is regular at $s=1$. Let

$$
r \log \zeta(s)=\sum_{n=1} \frac{d_{n}}{n^{s}}
$$

where

$$
d_{n}= \begin{cases}r / k & \text { if } n=p^{k} \\ 0 & \text { otherwise }\end{cases}
$$

Then by the classical estimate of Chebycheff,

$$
\sum_{n \leq x} d_{n}=\mathbf{O}\left(\frac{x}{\log x}\right)=\mathbf{o}(x)
$$

Consider

$$
g(s)=-\log \tilde{L}_{E}(s)-r \log \zeta(s)=\sum_{n=1}^{\infty} \frac{b_{n}}{n^{s}} .
$$

Since $r \log \zeta(s)+r \log (s-1)$ is regular at $s=1, g(s)$ is regular at $s=1$. Again by the previous lemma, $g(s)$ is convergent for $\Re(s)>1$. Now we apply Riesz's theorem 
On a Conjecture of Birch and Swinnerton-Dyer

above to the sequence $\lambda_{n}=\log n, y=\log x$. Then $g(s)$ satisfies all the conditions. Furthermore,

$$
B(x):=\sum_{n \leq x} b_{n}=\sum_{n \leq x}\left(\tilde{c}_{n}+d_{n}\right)=\mathbf{o}(x) .
$$

By the theorem, we know

$$
\sum_{n \leq x} \frac{\tilde{c}_{n}+d_{n}}{n}=A^{\prime}+\mathbf{o}(1)
$$

This implies

$$
C(x)=\sum_{n \leq x} \frac{\tilde{c}_{n}}{n}=\sum_{n \leq x} c_{n}=-\sum_{n \leq x} \frac{d_{n}}{n}+A^{\prime}+\mathbf{o}(1)=-r \log \log x+A+\mathbf{o}(1) .
$$

As noted in the remark at the end of Section 2, the asymptotic formula of $C(x)$ is equivalent to the B-SD conjecture. The proof is now complete.

Remark The condition

$$
\sum_{n \leq x} \tilde{c}_{n}=\tilde{C}(x)=\mathbf{o}(x)
$$

is much deeper than what we know at present. If we assume the Riemann hypothesis, we only can get $\tilde{C}(x)=\mathbf{O}(x \log x)$. Even if we assume the pair-correlation conjecture, we only have $\tilde{C}(x)=\mathbf{O}\left(x(\log x)^{1 / 2}\right)$. On the other hand, one can show $\tilde{C}(x)=$ $\Omega(x \log \log \log x / \log x)$. However, in [8], Montgomery gave a heuristic argument treating the error term occurring in classical prime number theory. When applied to our context, this suggests that $\tilde{C}(x)=\mathbf{O}\left(x(\log \log \log x)^{2} / \log x\right)$. Thus, it is likely that the $\mathrm{B}-\mathrm{SD}$ conjecture is true, from this perspective.

\section{An Oscillation Theorem}

Now we would like to know how the coefficient $c(x)$ in the conjecture behaves. In Birch's original paper, based on empirical data, it was noticed that $c(x)$ oscillates and it is hard to prove this directly. We cannot prove that $c(x)$ oscillates. However, we can prove $R(x)$, the remainder term of $C(x)$ (see Section $2,(\star))$ indeed oscillates.

We need the following oscillation theorem.

Theorem (Landau's Oscillation Theorem [1, Theorem 2]) If $\sum_{1}^{\infty} a_{n} n^{-s}$ is a Dirichlet series (respectively, $\int_{1^{-}}^{\infty} x^{-s} d F(x)$ a Mellin transform) that converges for $\Re(s)>\alpha$ and if the associated analytic function is regular at the point $s=\alpha$, then either the series (transform) converges to the left of the point $s=\alpha$ or else the sequence $a_{n}$ is not ultimately of one sign ( $F$ is not ultimately monotonic). Equivalently, if $a_{n}$ or $d F$ is of one sign from some point onward, then the abscissa of convergence exceeds $\alpha$ or $\hat{F}$ must have a singularity at the point $s=\alpha$. 
Now we can state our oscillation theorem.

Theorem 4 Assume the B-SD conjecture. Then $R(x)$ oscillates, i.e., $R(x)$ is not ultimately monotone.

Proof We know

$$
-\log \tilde{L}_{E}(s+1)=\sum \frac{c_{n}}{n^{s}}=\int_{1}^{\infty} \frac{1}{x^{s}} d C(x)
$$

Let

$$
G(s)=\int_{1}^{\infty} \frac{1}{x^{s}} d(r \log \log x-A)
$$

It is easy to see that

$$
G(s)=-r \log s+H(s),
$$

where $H(s)$ is regular for $\Re s \geq 1$ and $H(s)=\mathbf{O}(1)$. Let

$$
F(x)=r \log \log x-A+C(x)=R(x)
$$

We have

$$
-\log \tilde{L}_{E}(s+1)+G(s)=\int_{1}^{\infty} \frac{1}{x^{s}} d F(x) .
$$

Set $\alpha=0$ and apply Landau's theorem above on $F(x)$. We know that

$$
-\log \tilde{L}_{E}(s+1)+G(s)
$$

is regular at $s=0$. Since $\log \tilde{L}_{E}(s+1)$ has many singularities on the line $\Re(s)=0$, the function $\int_{1}^{\infty} x^{-s} d F(x)$ cannot converge to the left of $\Re(s)=0$. We conclude that $F(x)=R(x)$ is not ultimately monotonic.

In addition, we can say a little bit more about $R(x)$.

Proposition 5 For any $\epsilon>0$, the function $R(x)$ is $\Omega\left(1 /(\log x)^{1+\epsilon}\right)$.

Proof If the assertion is wrong, there exists $\epsilon>0$ such that

$$
R(x)=\mathbf{O}\left(\frac{1}{(\log x)^{1+\epsilon}}\right) .
$$

Then

$-\log \tilde{L}_{E}(s+1)=\int_{1}^{\infty} \frac{1}{x^{s}} d(-r \log \log x+A+r(x))=r \log s+H(s)+\int_{1}^{\infty} \frac{1}{x^{s}} d R(x)$.

Let

$$
J(s)=\int_{1}^{\infty} \frac{1}{x^{s}} d R(x) .
$$


On the line $\Re(s)=0$,

$$
\begin{aligned}
|J(s)|=\left|\int_{1}^{\infty} \frac{1}{x^{s}} d R(x)\right|=\left|s \int_{1}^{\infty} \frac{|R(x)|}{x} d x\right| & =\mathbf{O}\left(\int_{3}^{\infty} \frac{1}{x(\log x)^{1+\epsilon}} d x\right) \\
& =\mathbf{O}\left(\left.(\log x)^{-\epsilon}\right|_{3} ^{\infty}\right)=\mathbf{O}(1)
\end{aligned}
$$

Hence, $J(s)$ is regular on the line $\Re(s)=0$. This is impossible since $\log \tilde{L}_{E}(s+1)$ has many singularities on it. This is a contradiction and we have finished the proof.

We believe that the finer analysis of the zeros on the critical line can lead to a better $\Omega$ theorem of $R(x)$. We relegate this work for future research.

\section{References}

[1] P. T. Bateman and H. G. Diamond, On the oscillation theorems of Pringsheim and Landau. In: Number theory, Trends Math, Birkhäuser, Basel, 2000, pp. 43-54,

[2] B. Birch, Conjectures concerning elliptic curves. Proc. Symp. Pure Math, 8, Amer. Math. Soc, Providence, RI, 1965, pp. 106-112.

[3] C. Breuil, B. Conrad, F. Diamond and R. Taylor, On the modularity of elliptic curves over Q: wild 3-adic exercises. J. Amer. Math. Soc. 14(2001), 843-939.

[4] K. Conrad, Partial Euler products on the critical line. Canad. Math. J. 57(2005), 267-297.

[5] D. Goldfeld, Sur les produits eulériens attachés aux courbes elliptiques. C. R. Acad. Sci. Paris Sér. I Math. 294(1982), 471-474.

[6] Hans Arnold Heilbronn, The collected papers of Hans Arnold Heilbronn. John Wiley and Sons, New York, 1988, pp. 168-174.

[7] H. Iwaniec, Topics in classical automorphic forms. Graduate Studies in Mathematics, 17, American Math. Soc., Providence, RI, 1997.

[8] H. L. Montgomery, The zeta function and prime numbers. Queen's Papers in Pure and Appl. Math. 54(1980), 1-31.

[9] M. Riesz, Ein Konvergenzsatz für Dirichletsche Reihen. Acta Mathematica 40(1916), 350-354.

[10] A. Wiles, Modular elliptic curves and Fermat's last theorem. Ann. of Math. 141(1995), 443-551.

Department of Pure Mathematics University of Waterloo

Waterloo, ON

N2L $3 G 1$

e-mail:wtkuo@math.uwaterloo.ca
Department of Mathematics and Statistics, Queens University

Kingston, ON

K7L $3 N 6$

e-mail:murty@mast.queensu.ca 